\title{
The Dynamic Modell of Sleep Disorders - A Therapy-Tool for Cognitive Behavioral Therapy for Non-organic Sleep Disorders
}

\author{
Carolin Marx-Dick \\ Institute for Clinical Psychology, Technical University, Dresden, Germany
}

Email address:

c.marx-dick@schlafgut-dresden.de

\section{To cite this article:}

Carolin Marx-Dick. The Dynamic Modell of Sleep Disorders - A Therapy-Tool for Cognitive Behavioral Therapy for Non-organic Sleep Disorders. American Journal of Psychiatry and Neuroscience. Vol. 8, No. x1, 2020, pp. 6-11. doi: 10.11648/j.ajpn.20200801.12

Received: December 9, 2019; Accepted: December 25, 2019; Published: January 21, 2020

\begin{abstract}
The dynamic model of sleep disorders was carried out in the framework of the study of the dynamic of sleep disorders. On the basis of this circulation model, deficient sleep areas can be uncovered and a disturbance model can be developed together with the patients. Afterwards an individuell therapeutic rationale for breaking out of the "vicious circle" of sleep disturbances can be created. The study was a randomized controlled study. Test subjects suffered from non-organic insomnia. Also person suffering from psychological comorbidities were included. A total of 158 records were included in the calculations. The 5 expected factors "disturbed sleep", "fatigue during the day", "reduced performance", "worries" and "tension" could be extracted by factor analysis. Thus the "dynamic model of sleep disorders" could be mapped on the basis of the data of the SLEEP Quality Inventory. Overall, it can be said that all theoretically expected factors could be extracted on the basis of the factor analysis of the data. However, some factors showed weaknesses regarding a clean load of items. The following practical application of the model and the intervention showed that the model depicts only 2 levels of the behaviour and experience of the affected persons, the "psychophysiology and cognitions" as well as the "homeostatic principle". A third level of explication, "emotional dysregulation" has to be regarded in future.
\end{abstract}

Keywords: Insomnia, Sleep Disorders, Kognitive Behavioral Therapy, CBT-I, Dynamic Modell

\section{Introduction}

Non-organic sleep disorders, are very complex diseases that are often comorbid. They can have many different causes. They create a high level of suffering in those affected.

CBT-I is rightly the method of choice for the treatment of insomnia [1]. In order to be able to design these therapies even more effectively and above all more economically in the future, it is advantageous to recognise the individual causes and the resulting disease dynamics. In this way, the individual therapy components (also in group settings) can be selected more individually.

This requires a simple analysis scheme that is easy to understand, time-saving and can be used extensively in the everyday treatment (primary care) of doctors and therapists.

In the frame of the study "the dynamic of sleep disorders" the dynamic model of sleep disorders was developed. It represents the pathogenesis of non-organic sleep disorders and serves to create the bio-psycho-social condition model individually for each patient. The contents and individual factors of the model have evolved in the psychotherapeutic treatment practice of patients and a similar pattern has recurred. For the statistical validation of the factors and their dynamic interconnection, it was evaluated and checked for validity in the study described here.

In addition to primary insomnia, other forms of nonorganic sleep disorders such as non-organic hypersomnia and sleep-wake rhythm disorders can also be modelled in the dynamic model of sleep disorders. The development and maintenance of cormorbidities are also shown.

Figure 1 shows the "dynamic model of sleep disorders". Basically, a disturbed sleep is assumed because it represents the pathogenesis of non-organic sleep disorders. As already mentioned, various phenomena of dyssomnia (DSM F51) can be depicted here, since a homeostatic process is assumed as 
the basis. Thus, sleep changes caused by disturbances are possible in any direction.

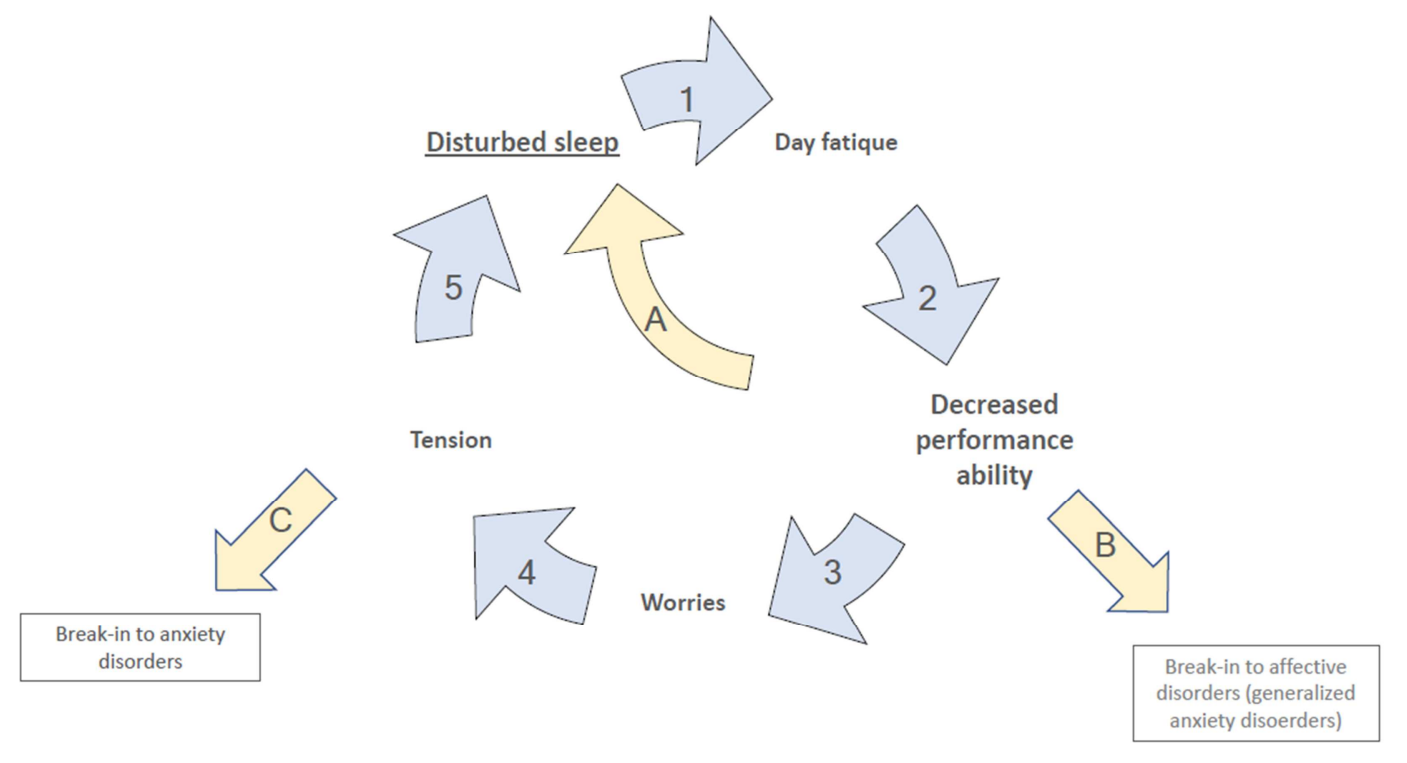

Figure 1. Dynamic model of sleep disorders.

Process 1: Disturbed sleep leads to daytime fatigue [2]. In contrast to evening fatigue, daytime fatigue is an undesirable condition. This is closely connected with exhaustion, cognitive performance losses, physical complaints such as headaches, gastrointestinal complaints, tension, dizziness, headaches, hallucinations, euphoria, concentration, attention and memory disorders as well as listlessness, lack of energy and drive [3].

Process 2: In the psychotherapeutic work, it turned out that daytime fatigue leads to arbitrary and involuntary protective behaviour. On the one hand, it is possible that those affected consciously spare themselves by sleeping longer at noon or in the morning, by having a longer bed period, by skipping or postponing important tasks and appointments [4]. Somnolence occurs consciously, but unintentionally, throughout the course of the day; it can cause a momentary sleep that can become very dangerous depending on the current activity [5]. It is also known today that some major catastrophes and accidents occurred during the night shift, e.g. the reactor explosion in Chernobyl in 1986 or the chemical-gas catastrophe in Bophal in 1984 [6].

Process A: Also in mental work a fatigue effect sets in after a certain duration and strain. Reasons for mental fatigue are still not known, it may have disappeared in a flash with a change of tasks [7]. In addition, the individual biological rhythm, age and duration of sleep deprivations have an influence on cognitive performance [8]. Cognitive activities are also associated with increased oxygen and glucose consumption, but this is primarily due to increased muscle tone. Depending on the type of cognitive task, various physiological parameters such as heart rate, blood pressure, pulse, body temperature, respiratory rate, etc. increase or decrease [9]. There is therefore a close connection between physiology and the human psyche, they are mutually dependent [10), [11]. By reducing or omitting physical and psychological stress, the lack of fatigue due to the lack of cognitive stress due to a lack of cognitive performance does not build up sufficient sleep pressure until the desired time to fall asleep.

Process 3: On the other hand, a lack of performance and the protective behaviour leads to insufficiencies in many areas and thus to worries [12]. Those affected often have the feeling that they are insufficient in their everyday lives and are no longer able to perform important professional and private tasks satisfactorily. This leads to worries about the relationship with the partner and with the children, to worries about the professional situation and fear of a possible warning or dismissal. In the evening, sleepless in bed, these worries can germinate well by forming strong synaptic connections through Rehersal. The person gets used to pondering in bed instead of sleeping. This means that lying in bed is closely linked to worrying by means of operant conditioning, instead of relaxation and sleep.

Process B: At this point a break-in into a depressive or anxious symptomatology is very obvious, as the affected persons increasingly experience not being able to perform well and experience little self-efficacy. The process of worrying or permanent exhaustion is often accompanied by social withdrawal. This means that those affected have to accept losses. Well empirically tested models that include this are the amplifier-loss model [13], the model of learned helplessness according to Seeligman [14] or the psychophysiological model of fear [15].

Process 4: While Thomas Borkovec and his colleagues were investigating the properties and treatment of insomnia in the 1970s, they came across the phenomenon of "worrying about oneself". They discovered that worrying about oneself leads to physical tension, some of which is not even noticed by those affected. This tension ultimately prevents them from falling asleep. The worries come automatically, unintentionally and almost force themselves upon them [1618]. A relaxed awake state with alpha activity precedes the 
sleep phase N1 [19]. If this is not possible, no falling asleep and no transition into deeper sleep phases is possible.

Process C: A repeated or permanent worries and worries process causes a chronically increased muscle tone in those affected. This in turn is a good breeding ground for the development of anxiety disorders [20]. The often comorbid generalised anxiety disorders, specific phobias in front of the bed, general stress disorders, panic and other anxiety disorders are explained here in the dynamic model.

All these processes lead to disturbed sleep, which can express itself with various symptoms: Sleep disturbances, early waking, hypersomnia, nightmares and a fundamentally disturbed sleep-wake rhythm.

With the dynamic model of sleep disorders, different aspects of sleep disorders can be considered and explained individually:

1. The causes: Why does the affected person have sleep disorders? It can therefore be used to create a biopsycho-social condition model.

2. The effects: Which areas of the affected person's everyday life are affected by the sleep disorder?

3. Selected aspects of the biological basics: To what extent does the sleep disorder interfere with biological and physiological rhythms?

4. The individual symptoms: Which symptoms are caused by the sleep disorder?

5. The behaviour: How does the affected person behave due to his sleep disorder? How does he maintain it?

6. The therapy approaches: At which point can the affected person break through this dynamic in order to sleep again restfully and create a fresh and rested everyday life.

The dynamic model is used to uncover individual dysfunctional areas of life, such as dysfunctional beliefs about sleep or sleep-deprived behaviour. Together with the patients an individual disturbance model is developed. The attending physician or therapist can use this model to develop the therapy rationale to be used and thus specifically select individual treatment techniques. These fit exactly into the everyday life of the respective patient and holistically treat the entire disorder, sleep disorder and existing comorbidities in one go [21].

In summary it can be said that for each patient individually his own dynamic model is developed as a "tool" to illustrate his very individual symptoms, to uncover dysfunctional ways of thinking and behaving and to restructure them in close cooperation with the patient. It is then used in cognitive behavioural therapy and directly maps therapy effects.

\section{Methods}

The data for the model test were collected in the context of the randomized, controlled, clinical intervention vs. control waiting group study "the dynamics of sleep disorders". Basically, this study evaluated a newly adapted, specific and individualizable cognitive-behavioural therapeutic manual for the therapy of non-organic sleep disorders [22].

The recruitment of the volunteers was carried out by the Clinic and Polyclinic for Psychiatry at the University Hospital Carl Gustav Carus Dresden. All volunteers were included who met the diagnostic criteria for (nonorganic/primary) insomnia (ICD 10, DSM IV) and subjectively suffered from sleep problems and not restful sleep. Subjects with comobidities were also included in the study in a controlled manner. The practical relevance of this study was the basic innovation factor of this study, as more than $70 \%$ of the patients suffer from mental comorbidities [23].

158 test person answered the Sleep Quality Inventory (SLEEP QUI) which was specifically developed for this study [22]. To create the Sleep QUI, the already established questionnaire "Pittsburgh Sleep Quality Index" [24] was included. In addition, numerous non-validated questionnaires, which are usually used by sleep laboratories for patient surveys, have been included. Based on this data and the underlying model of the dynamics of sleep disorders, the Sleep QUI was generated.

For the replication of the factors of the model, a factor analysis was calculated taking into account the theoretically and content consistent items. Additionally individual factors were evaluated using descriptive statistics and their interrelationships were examined using contingency tables and chi-square tests.

\section{Results}

The individual factors of the "dynamic model of sleep disorders" were extracted by means of a factor analysis of the relevant items of the questionnaire. The results of the factor analysis are presented in Table 1.

Table 1. Results of Factor Analysis for Model Replication.

\begin{tabular}{|c|c|c|c|c|c|}
\hline \multirow{2}{*}{ Variable } & Factor 1 & Factor 2 & Factor 3 & Factor 4 & Factor 5 \\
\hline & Disturbed sleep & Day fatique & decreased performance ability & Worries & tension \\
\hline Sleep disturbance & 830 & & & & \\
\hline Sleep latency & ,862 & & & & \\
\hline Sleep-through disorder & ,494 & & &,- 441 & \\
\hline Early awakening &, 431 & & &,- 594 & \\
\hline Unwanted fatigue & & & & ,507 & \\
\hline Body symptoms in the morning & & ,752 & & & \\
\hline Body symptoms during the day & & ,813 & & & \\
\hline Pain & & ,630 & & & ,410 \\
\hline Conscious protection in general & & & ,764 & & \\
\hline Conscious protection by pauses & & &, 843 & & \\
\hline
\end{tabular}




\begin{tabular}{|c|c|c|c|c|c|}
\hline \multirow{2}{*}{ Variable } & Factor 1 & Factor 2 & Factor 3 & Factor 4 & Factor 5 \\
\hline & Disturbed sleep & Day fatique & decreased performance ability & Worries & tension \\
\hline $\begin{array}{l}\text { Conscious protection through } \\
\text { transformation }\end{array}$ & & &, 549 & ,453 & \\
\hline Lunch nap & & & ,728 & & \\
\hline Unconscious protection & & & &, 592 & \\
\hline Cognitive deficits & & & & ,461 & \\
\hline Fears/anxious thoughts & & & &, 802 & \\
\hline Increased body tension & & & & & ,797 \\
\hline Thoughts before going to bed & & & & & 692 \\
\hline
\end{tabular}

Extraction method: principal component analysis. Rotation method: Varimax with Kaiser normalization, the rotation is converged in 9 iterations. All values $<.400$ were hidden for clarity; dark blue background: Factor loads according to theory; light blue background: Factor charges that can be interpreted.

In total, the 5 expected factors "disturbed sleep", "fatigue during the day", "reduced performance", "worries" and "tension" could be extracted with a variance clarification of $70 \%$.

This is very clear for the initial factor 1 "disturbed sleep". All items included on a theoretical basis: "sleep disturbance" with the "sleep latency", "sleep disturbance" and "early awakening", loaded cleanly on this factor.

The factor 2 "daytime sleepiness" could also be mapped, but not so clearly. The theoretical basis and considerations that "daytime fatigue" on the one hand occurs with subjectively noticeable fatigue and various body symptoms such as nausea, headaches, dizziness, etc. could not be clearly extracted. The body symptoms and the pain were cleanly loaded on this factor. The subjectively perceived daytime tiredness, however, was based on factor 4 "worries".

The factor 3 "reduced performance" could also be extracted. The items "conscious protection", "conscious protection through breaks", "conscious protection through reshaping" and "midday nap" showed high correlations. The theoretically expected items "unconscious protection" and "cognitive deficits", on the other hand, charged factor 4 "worries".

Factor 4 "worries" could not be depicted as clearly as the other factors. As expected, the theoretically expected items "fears/anxious thoughts" and "thoughts" showed high correlations. In addition, however, the items "daytime fatigue", "unconscious rest" and "cognitive deficits" also loaded on this factor.

Factor 5 "Tension" on the other hand could be extracted more clearly again. The expected items "tension" and "thoughts" before going to bed clearly loaded on this factor. In addition, the item "pain" loaded on this factor, but this can also be justified theoretically.

\section{Discussion}

Overall, it can be said that all theoretically expected factors could be extracted on the basis of the factor analysis of the data. Individual items did not load on the expected factor, but this can be explained on a theoretical basis.
Since this is a dynamics model, it is assumed that one factor determines the next. Thus, individual symptoms can pass to the other factor as part of the emergence and maintenance of the sleep disorder, thus favouring the occurrence of this factor and keeping the dynamics going.

In addition to the robust representations of the factors, however, there were items that loaded differently than the underlying theory.

For example, the item "unwanted fatigue" loaded on the factor "worries" instead of "daytime fatigue". One reason for this could be that sleep is a main regulator for many systems, such as affectivity and the associated automatic assessment of situations or symptoms [25]. "Unwanted fatigue" is therefore a factor that patients automatically evaluate negatively. It is therefore likely that there is a relationship between "unwanted fatigue" and "worry" [26].

Daytime fatigue is therefore closely related to body symptoms [27]. People with sleep disorders show fundamentally higher rates of somatoform symptoms than those without insomnia. Pain is also clearly correlated with poor sleep quality [28]. Furthermore, depression symptoms are more frequent in the context of daytime sleepiness. These also correlate strongly with mood, appetite and selfassessment [29].

Unwanted fatigue causes worries, because people are then not so efficient or do not feel efficient and worry about their fatigue itself [16]. As expected, the items sleep disturbances and early awakening loaded on the factor disturbed sleep and at the same time negatively on the factor worries. This would mean that people suffering from sleep disturbances and early waking would worry less. This is contrary to the theory that when people wake up at night or too early, they worry more about not falling asleep again $[31,4]$.

As expected, the pain items were also a physical symptom of the day and were also a factor of tension. This is also theoretically justified, since tension can aggravate and even cause pain [31]. Therefore, tension very often occurs in combination with pain.

There are numerous studies that show that the perception of pain is significantly increased after just one night of poor sleep quality $[32,28]$.

It has been assumed that the items for unconscious protection and cognitive deficits are based on the factor of reduced performance. Instead, they loaded on the factor worries. This means that unconscious protective behavior and noticing cognitive deficits also lead to worries. People who suffer from sleep disorders report less activity in their free 
time than people with good sleep quality [33]. This applies to the intensity of the activity as well as the duration and frequency. These results were found in both sedentary workers, who are more likely to have cognitive performance, and physical workers.

This thus represents the close interconnectedness and dynamics between the individual factors.

\section{Acknowledgements}

The model was developed under consideration of many years of experience and systematic collection of reports of sleep disturbed patients. This study is a first attempt to evaluate the dynamic model of non-organic sleep disorders, not only insomnia. With the intervention based on this model, in the form of a cognitive behavioral therapy against sleep disorders, this model should be made usable. This means that the entire study was designed to map this dynamic, the SLEEP QUI was designed to map the factors, and the intervention [22] was composed accordingly. The above described measures have now been applied in a broad spectrum of practice. Weaknesses were found with regard to the consideration of the emotionality of people with sleep disorders. In addition to the physical underactivation and psychophysiological overactivation described above, sympathetic-activating phenomena should also be taken into account. The basic emotion "anger/rage" was particularly noticeable in some disease dynamics. Therefore, the "dynamic model of sleep disorders" was extended to a 3level model of the dynamics of sleep disorders. Here we distinguish between "psychophysiology and cognitions", "homeostatic principle" and "emotional dysregulation". "Psychophysiology and cognitions" have already been described in the "dynamic model of sleep disorders" with processes 1 to 5 . The "homeostatic principle" found its illustration in the processes A, B and C. The "homeostatic principle" was described in the "dynamic model of sleep disorders" with the processes 1 to 5 . Consequently, level 3 "emotional dysregulation" was missing in the "dynamic model of sleep disorders".

In a further study, the 3-level model of the dynamics of sleep disorders is evaluated and checked for validity in a larger study at the Centre for Healthy Sleep in Dresden.

\section{References}

[1] Van der Zweerde T, Bisdounis L, Kyle SD, Lancee J, Van STraten A. Cognitive behavioral therapy for insomnia: A meta-analysis of long-term effects in controlled studies. Sleep Med Rev. 2019 Aug 12; 48: 101208.

[2] Kim SJ, Kim S, Jeon S, Leary EB, Barwick F, Mignot E. Factors associated with fatigue in patients with insomnia. $\mathrm{J}$ Psychiatr Res. 2019 Oct; 117: 24-30.

[3] Achermann P, Borbély AA. Simultation of daytime vigilance by the additive interaction of a homeostatic and circadian process. Biol Cybern. 1994; 71: 115-21.
[4] Riemann D, Spiegelhalder K, Vorderholzer U, Kaufmann R, Seer N, Klöpfer C, Hornyak M, Espie C, Perlis M. Primäre Insomnien: Neue Aspekte der Diagnostik und Differentialdiagnostik, Äthiologie und Pathophysiologie sowie Psychotherapie. Somnologie. 2007. 11: 57-71.

[5] Findley LJ, Fabrizzio M, Toni G, Suratt P. Automobile crashes in patients with obstructive sleep apnea. Am Rev Respir Dis. 1988. 147: 56.

[6] Knauth P. Schichtarbeit, Nachtarbeit. In: Triebig G, Kentner M, Schiele R (Hrsg). Arbeitsmedizin. Handbuch für Theorie und Praxis. Gentner, Stuttgart, 2003. S. 733-742.

[7] Doran SM, Van Dongen HP, Dinges DF. Sustained attention performance during sleep deprivation: evidence of state instability. Arch Ital Biol. 2001 Apr; 139 (3): 253-67.

[8] Silva EJ, Wang W, Ronda JM, Wyatt JK, Duffy JF. Circadian and wake-dependent influences on subjective sleepiness, cognitive throughput, and reaction time performance in older and young adults. Sleep. 2010 Apr; 33 (4): 481-90.

[9] Wyatt JK, Ritz-De Cecco A, Czeisler CA, Dijk DJ. Circadian temperature and melatonin rhythms, sleep, and neurobehavioral function in humans living on a $20-\mathrm{h}$ day. Am J Physiol. 1999 Oct; 277 (4 Pt 2): R1152-63.

[10] Rohmert W. 1973. In: Schmidtke H (Ed.). Ergonomie 1. Hanser, München, S. 225-255.

[11] Dawson D, Chapman J, Thomas MJ. Fatigue-proofing: a new approach to reducing fatigue-related risk using the principles of error management. Sleep Med Rev. 2012; 16: 167-75.

[12] Borkovec TD, Shadick RN, Hopkins M. The nature of normal and pathological worry. In Rapee RM, Barlow DH (Eds.). Chronic Anxiety: Generalized anxiety disorder and mixed anxiety-depression. 1991; New York: Guilford Press.

[13] Lewinsohn PM. A behavioral approach to depression. In Friedman RJ, Katz MM (Eds.). The psychology of depression: Contemporary theory and research. 1974; New York: John Wiley \& Sons.

[14] Seeligman MEP. Helplessness: On depression, development, and death. 1975; San Francisco: Freeman.

[15] Ehlers A, Margraf J. Agoraphobien und Panikanfälle. In H. Reinecker (Hrsg.), Lehrbuch der Klinischen Psychologie. Modelle psychischer Störungen (S. 73-106). 1990; Göttingen: Hogrefe.

[16] Borkovec TD, Hennings BL. The role of physiological attention-focusing in the relaxation treatment of sleep disturbance, general tension, and specific stress reaction. Behav Res Ther. 1978; 16 (1): 7-19.

[17] Borkovec TD. Insomnia. J Consult Clin Psychol. 1982; 50 (6): 880-895.

[18] Borkovec TD, Castello E. Efficacy of applied relaxation and cognitive-behavioral therapy in the treatment of generalized anxiety disorder. J Consult Clin Psychol. 1993; 61 (4): 611619.

[19] Stuck BA, Mauerer JT, Schredl M, Weeß HG. Praxis der Schlafmedizin. Springer, Heidelberg; 2009.

[20] Pluess M, Conrad A, Wilhelm FH. Muscle tension in generalized anxiety disorder: a critical review of the literature. J Anxiety Disord. 2009; Jan; 23 (1): 1-11. 
[21] Marx C. Nichtorganische Schlafstörungen. Berlin: Springer; 2016.

[22] Marx C. Die Dynamik der Schlafstörungen. Dissertation. 2016.

[23] Roth T. Insomnia: Definition, Prevalence, Etiology, and Consequences. J Clin Sleep Med 2007; 3 (Suppl 5): 7-10.

[24] Buysse DJ, Reynold CF, Monk TH, Berman SR, Kupfer DJ. 1989. The Pittsburgh Sleep Quality Index: a new instrument for psychiatric practice and research. Psychiatry Res 28 (2): 193-213.

[25] Palagini L, Bastien CH, Marazziti D, Ellis JG, Riemann D. The key role of insomnia and sleep loss in the dysregulation of multiple systems involved in mood disorders: A proposed model. J Sleep Res. 2019 Dec; 28 (6): e12841. doi: 10.1111/jsr.12841. Epub 2019 Apr 10.

[26] Veale D, Poussin G, Benes F, Pepin JL, Levy P. Identification of quality of life concerns of patients with obstructive sleep apnoea at the time of initiation of continuous positive airway pressure: a discourse analysis. Qual Life Res. 2002 Jun; 11 (4): 389-99.

[27] Cathébras PJ, Robbins JM, Kirmayer LJ, Hayton BC. Fatigue in primary care: prevalence, psychiatric comorbidity, illness behavior, and outcome. J Gen Intern Med. 1992 May-Jun; 7 (3): 276-86

[28] Johnson KA, Gordon CJ, Grunstein RR. Somatic symptoms are associated with Insomnia disorder but not Obstructive Sleep Apnoea or Hypersomnolence in traumatic brain injury. Neuro Rehabilitation. 2019 Nov 28. doi: 10.3233/NRE192868 [Epub ahead of print].

[29] Ji X, Bastien CH, Ellis JG, Hale L, Grandner MA. Disassembling insomnia symptoms and their associations with depressive symptoms in a community sample: the differential role of sleep symptoms, daytime symptoms, and perception symptoms of insomnia. Sleep Health. 2019 Aug; 5 (4): 376381. doi: 10.1016/j.sleh.2018.12.009. Epub 2019 Mar 28.

[30] Crönlein T. Schlafstörungen: Ein Gruppentherapieprogramm für den stationären Bereich. Göttingen: Hogrefe; 2013.

[31] Hampton AJ, Hadjistavropoulos T, Gangon MM, Williams J, Clark D. The effects of emotion regulation strategies on the pain experience: a structural laboratory investigation. Pain; 2015; 156 (5): 868-879.

[32] Whibley D, Braley TJ, Kratz AL, Murphy SL. Transient Effects of Sleep on Next-Day Pain and Fatigue in Older Adults With Symptomatic Osteoarthritis. J Pain. 2019 Nov; 20 (11): 1373-1382. doi: 10.1016/j.jpain.2019.04.011. Epub 2019 May 11.

[33] Bláfoss R, Sundstrup E, Jakobsen MD, Bucht H, Garde AH, Andersen LL. Are Insomnia Type Sleep Problems Associated With a Less Physically Active Lifestyle? A Cross-Sectional Study among 7,700 Adults From the General Working Population. Front Public Health. 2019 May 14; 7: 117. doi: 10.3389/fpubh.2019.00117. eCollection 2019. 